\title{
A Mathematical Model of Glucose-Insulin Interaction with Time Delay
}

\section{Saber $\mathbf{S}^{1 *}$, Bashier EBM ${ }^{2}$, Alzahrani $\mathbf{S M}^{3}$ and Noaman IA $^{3}$}

${ }^{1}$ Mathematics Department, Faculty of Science and Arts, Baljurashi, Albaha University, Saudi Arabia

${ }^{2}$ Department of Applied Mathematics, Faculty of Mathematical Sciences, University of Khartoum, Sudan

${ }^{3}$ Faculty of Arts and Science, Al-Baha University, Almandaq, Saudi Arabia

\begin{abstract}
This paper extends the work by incorporating a time delay in the interaction term of the glucose and insulin. The new model is analyzed and the stability properties have been concluded. Numerical simulations are carried out to confirm the theoretical results.
\end{abstract}

Keywords: Glucose-Insulin; Stability; Diabetes; Equilibrium point; Asymptotically

\section{Introduction}

Body cells need energy to function, and glucose is the main source of energy for the cells in the living organisms [1]. Glucose comes from carbohydrate food after a meal intake. Therefore, the glucose level increases after food intake [2-4]. Here comes the role of the Insulin, which is a hormone that is produced by the Pancreas for the purpose of regulating the Glucose concentration in the blood [5-8]. The main function of the Insulin is to help the body cells to absorb the free blood glucose [9-11]. After its release by the Pancreas, the Insulin attaches with the cells and signals them to absorb the sugar from the blood stream [12-14].

Generally, two types of failure in controlling the level of sugar in the blood can occur. The first failure type happens when the Pancreas fails to produce enough amount of insulin [15]. This failure type is referred to as juvenile diabetes. The second failure type happens when the body cells fail to respond to insulin in a proper way [16]. The diabetes mellitus includes diabetes of types 1 and $2[16,17]$.

According to the International Diabetes Federation (IDF), 387 million people have diabetes in 2014, and this number will rise to 592 million by 2035. The number of people with diabetes of type 2 is increasing in every country of the world. Moreover, Diabetes caused 4.9 million deaths in 2014; every seven seconds a person dies from diabetes (http://www.idf.org/diabetesatlas/update-2014).

The modeling of the glucose-insulin system has become an interesting topic, where, many mathematical models were developed to understand and predict the Glucose-Insulin dynamics [1,9,11,12,17]. Among the most widely used models to study diabetes dynamics, is the minimal model which is used in the interpretation of the intravenous glucose tolerance test [5]. In this paper, we extend the work [9] by considering a time delay. We propose the following general model for the interaction of glucose and insulin

$$
\begin{aligned}
& \frac{d x}{d t}=-a_{1} x(t)-a_{2} x(t-\tau) y(t-\tau)+a_{3}, t \in[0, T], \\
& \frac{d y}{d t}=b_{1} x(t)-b_{2} y(t), t \in[0, T] .
\end{aligned}
$$

with initial data:

$$
\begin{aligned}
& x(\theta)=\phi(\theta), \theta<0, \\
& y(\theta)=\psi(\theta), \theta<0
\end{aligned}
$$

where $x \geq 0, y \geq 0, x$ represents glucose concentration, $y$ represents insulin concentration, $a_{1}$ is the rate constant which represents insulinindependent glucose disappearance, $a_{2}$ is the rate constant which represents insulin-dependent glucose disappearance, $a_{3}$ is the glucose infusion rate $b_{1}$ is the rate constant which represents insulin production due to glucose stimulation, $b_{2}$ is the rate constant which represents insulin degradation and the time delay $\tau$ represents the time taken by Pancreas to respond to the feedback of the glucose level.

The rest of this paper is organized as follows. We discuss the qualitative analysis of the model behavior. The description of the numerical method for solving the proposed model is introduced. Then, we show the behavior of the model for different values of the time delay. Finally, these conclusions are disused.

\section{Stability Analysis}

In this we discuss the qualitative analysis of the model behavior. We shall now investigate the dynamics of the delay system eqn. (1) The steady state or equilibrium (fixed point) of the system eqn. (1) is one of which

$$
\begin{aligned}
& x(t)=x(t-\tau)=x(0)=x^{*} \forall t, \\
& y(t)=y(t-\tau)=y(0)=y^{*} \forall t
\end{aligned}
$$

and as a consequence all the time derivatives vanish identically. Hence substituting

$$
\begin{aligned}
& x(t)=x(t-\tau) \text { and } \dot{x}=0, \\
& y(t)=y(t-\tau) \text { and } \dot{y}=0,
\end{aligned}
$$

in eqn. (1), it is easy to see that eqn. (1) has the equilibrium point $\left(x^{*}, y^{*}\right)$ as the following:

$$
\begin{aligned}
& x^{*}=\frac{-a_{1} b_{2}+\sqrt{\left(a_{1} b_{2}\right)^{2}+4 a_{2} b_{2} a_{3} b_{1}}}{2 b_{1} a_{2}}, \\
& y^{*}=\frac{-a_{1} b_{2}+\sqrt{\left(a_{1} b_{2}\right)^{2}+4 a_{2} b_{2} a_{3} b_{1}}}{2 b_{2} a_{2}} .
\end{aligned}
$$

${ }^{*}$ Corresponding author: Saber S, Mathematics Department, Faculty of Science and Arts, Baljurashi, Albaha University, Saudi Arabia, Tel: +966 17727 4111; E-mail: sayedkay@yahoo.com

Received September 03, 2018; Accepted September 12, 2018; Published September 19, 2018

Citation: Saber S, Bashier EBM, Alzahrani SM, Noaman IA (2018) A Mathematica Model of Glucose-Insulin Interaction with Time Delay. J Appl Computat Math 7 : 416. doi: 10.4172/2168-9679.1000416

Copyright: ( 2018 Saber S, et al. This is an open-access article distributed unde the terms of the Creative Commons Attribution License, which permits unrestricted use, distribution, and reproduction in any medium, provided the original author and source are credited. 
The interior-equilibrium point $E_{1}=\left(x^{*}, y^{*}\right)$ exists unconditionally as $x^{*}$ and $y^{*}$ are always positive as all the parameters are considered positive.

To linearize the model about the equilibrium point $E_{1}$ of model eqn. (1), let $u(t)=x(t)-x^{*}, v(t)=y(t)-y^{*}$. We then obtain the linearized model

$$
\begin{aligned}
& \frac{d u}{d t}=-a_{1} u(t)-a_{2} y^{*} u(t-\tau)-a_{2} x^{*} v(t-\tau)-a_{2} u(t-\tau)-a_{1} x^{*}-a_{2} x^{*} y^{*}+a_{3}, \\
& \frac{d y}{d t}=b_{1} u(t)-b_{2} v(t)+b_{1} x^{*}-b_{2} y^{*} .
\end{aligned}
$$

After removing nonlinear terms, we obtain the linear vibrational system, by using equilibria conditions as

$$
\begin{aligned}
& \frac{d u}{d t}=-a_{1} u(t)-a_{2} y^{*} u(t-\tau)-a_{2} x^{*} v(t-\tau), \\
& \frac{d y}{d t}=b_{1} u(t)-b_{2} v(t) .
\end{aligned}
$$

From the linearized model we obtain the characteristic equation

$\Delta(\lambda, \tau)=\lambda^{2}+a \lambda+(b \lambda+c) e^{-\lambda \tau}+d=0$,

where $a=a_{1}+b_{1}, b=a_{2} y^{*}, c=a_{2} b_{1} x^{*}+a_{2} b_{2} y^{*}$ and $d=a_{1} b_{2}$.

For $\tau=0$, the characteristic eqn. (6) becomes

$$
\Delta(\lambda, 0)=\lambda^{2}+(a+b) \lambda+c+d=0,
$$

Now, sum of the roots $=-(a+b)$ and product of the roots $=c+d$. Thus, we can say that both the roots of eqn. (7) are real and negative or complex conjugate with negative real parts if and only if

$$
a+b>0 \text { and } c+d>0 .
$$

Hence, in the absence of time delay, the equilibrium point $E_{1}$ is locally asymptotically stable if and only if both conditions $a+b>0$ and $c+d>0$ hold simultaneously.

Following [9]; The interior-equilibrium point $E_{1}$ is locally asymptotically stable when $\tau=0$ if

$\left(b_{1-} a_{2} x^{*}\right)^{2<4} b_{2}\left(a_{1+} a_{2} y^{*}\right)$.

Now for $\tau \neq 0$, if $\lambda=i \omega(\omega>0)$ is a root for the characteristic eqn. (6), then $\omega$ should satisfy the following equations

$$
\begin{aligned}
& \omega b \sin \omega \tau+c \cos \omega \tau=\omega^{2-}, \\
& \omega b \cos \omega \tau-c \sin \omega \tau=-a \omega,
\end{aligned}
$$

Which implies that

$$
\omega^{4}+\left(a^{2}-b^{2}-2 d\right) \omega^{2}+d^{2}-c^{2}=0 .
$$

Then we obtain

$$
\omega=\left(-\left(a^{2}-b^{2}-2 d\right) \pm \sqrt{\left(a^{2}-b^{2}-2 d\right)^{2}-4\left(d^{2}-c^{2}\right) / 2^{1 / 2}}\right.
$$

From the eqn.(11), it follows that if

$$
a^{2}-b^{2}-2 d>0 \text { and } d^{2}-c^{2>0}
$$

then eqn. (10) does not have any real solutions. From eqn. (11), we see that there is a unique positive solution $w_{0}^{2}$ if

$$
\begin{aligned}
& c^{2}-d^{2}<0 \\
& \text { If } d^{2}-c^{2>} 0, b^{2}-a^{2}-2 d>0, \text { and } \\
& \left(b^{2}-a^{2-} 2 d\right)^{2>} 4\left(d^{2-} c^{2}\right)
\end{aligned}
$$

hold, then there are two positive solutions $\omega_{ \pm}^{2}$.
To find the necessary and sufficient conditions for nonexistence of delay induced instability, we now use the following theorem.

\section{Theorem 1.1}

A set of necessary and sufficient conditions for $E_{1}$ to be asymptotically stable for all $\tau \geq 0$ is the following.

1. The real parts of all the roots of $\Delta(\lambda, 0)=0$ are negative [10].

For all real $m$ and $\tau \geq 0, \Delta(i \omega, \tau) \neq 0, i=\sqrt{-1}$.

\section{Theorem 1.2}

If conditions eqn.(8) and eqn.(12) and Theorem 1.1 are satisfied, then the equilibrium $E_{1}$ is asymptotically stable for all $\tau<\tau_{0}$ and unstable for $\tau>\tau_{0}$. Furthermore, as $\tau$ increase through $\tau_{0}$, E $E_{1}$ bifurcates into small amplitude periodic solutions, where $\tau_{0}=\tau_{0 n}$ as $n=0$.

Proof: For $\tau=0, E_{1}$ is asymptotically stable if condition eqn. (8) holds. Hence, by Butler's lemmma, $E_{1}$ remains stable for $\tau<\tau_{0}$. We now have to show that

$$
\left.\frac{d(\operatorname{Re} \lambda)}{d \tau}\right|_{\tau=\tau_{0}, w=w_{0}}>0 \text {. }
$$

This will signify that there exists at least one eigenvalue with positive real part for $\tau>\tau_{0}$. Moreover, the conditions of Hopf bifurcation [8] are then satisfied yielding the required periodic solution.

Substituting $w_{0}^{2}$ into eqn.(9) and solving for $\tau$, we get

$$
\tau_{0 n}=\frac{1}{w_{0}} \arctan \left(-\frac{w_{0}\left(a c-b d+b w_{0}^{2}\right)}{a b w_{0}^{2}+\left(d-w_{0}^{2}\right) c}\right)+\frac{2 n \pi}{w_{0}}, n=0, \pm 1, \pm 2, \ldots
$$

Substituting $\omega_{ \pm}^{2}$ into eqn.(9) and solving for $\tau$, we obtain

$$
\tau_{k}^{\mp}=\frac{1}{w_{ \pm}} \arctan \left(-\frac{w_{ \pm}\left(a c-b d+b w_{ \pm}^{2}\right)}{a b w_{ \pm}^{2}+\left(d-w_{ \pm}^{2}\right) c}\right)+\frac{2 k \pi}{w_{ \pm}}, k=0,1,2, \ldots .
$$

Differentiating eqn. (6) w.r.t. $\tau$, we obtain

$$
\left[2 \lambda+a+b e^{-\lambda \tau}-\tau(b \lambda+c) e^{-\lambda \tau}\right] \frac{d \lambda}{d \tau}=\lambda e^{-\lambda \tau}(b \lambda+c)
$$

thus

$$
\left(\frac{d \lambda}{d \tau}\right)^{-1}=\frac{2 \lambda+a}{-\lambda\left(\lambda^{2}+a \lambda+c\right)}+\frac{b}{\lambda(b \lambda+c)}-\frac{\tau}{\lambda}
$$

and by using

$$
e^{-\lambda \tau}=-\left(\frac{\lambda+a \lambda+d}{b \lambda+c}\right)
$$

we obtain

$$
\operatorname{sign}\left\{\frac{d(\operatorname{Re} \lambda)}{d \tau}\right\}_{\lambda=-w}=\operatorname{sign}\left\{\operatorname{Re}\left(\frac{d \lambda}{d \tau}\right)^{-1}\right\}=\operatorname{sign}\left[\frac{2\left(w^{2}-d+a^{2}\right)}{\left(-w^{2}+d\right)^{2}+a^{2} w^{2}}-\frac{b^{2}}{c^{2}+b^{2} w^{2}}\right]
$$

It follows that

$$
\left.\frac{d(\operatorname{Re} \lambda)}{d \tau}\right|_{\tau=\tau_{0}, w=w_{0}}>0 .
$$

Thus, the transversability condition holds, and hence, Hopf bifurcation occurs at $\tau=\tau_{0}$. This completes the proof.

\section{Theorem 1.3}

Let $\tau k \pm$ be defined in eqn.(16). If in eqns. (8) and (14) are satisfied, then the equilibrium point $E_{1}$ is stable when and unstable when, for some 
positive integer $m$. Thus, there are bifurcations at the equilibrium point $E_{1}$ when $\tau=\tau_{k}^{ \pm}, k=0,1,2, \ldots$

Proof: If conditions in eqn. (8) and (14) are satisfied, then to prove the theorem we need only to verify the transversability conditions $[6,7]$.

$\left.\frac{d(\operatorname{Re} \lambda)}{d \tau}\right|_{\tau=\tau_{k}^{ \pm}}>0$ and $\left.\frac{d(\operatorname{Re} \lambda)}{d \tau}\right|_{\tau=\tau_{k}^{-}}<0$

From eqn. (19), it follows that

$\operatorname{sign}\left\{\frac{d(\operatorname{Re} \lambda)}{d \tau}\right\}_{\lambda=i w_{+}}=\operatorname{sign}\left\{\frac{\sqrt{\left(b^{2}-a^{2}+2 d\right)^{2}-4\left(d^{2}-c^{2}\right)}}{\left\{\left(-\mathrm{w}_{+}^{2}+\mathrm{d}\right)^{2}+\mathrm{a}^{2} \mathrm{w}_{+}^{2}\right\}\left\{\mathrm{c}^{2}+\mathrm{b}^{2} \mathrm{w}_{+}^{2}\right\}}\right\}$

Thus

$\left.\frac{d(\operatorname{Re} \lambda)}{d \tau}\right|_{\tau=\tau_{k}^{+}, w=w_{+}}>0$

Again,

$\operatorname{sign}\left\{\frac{d(\operatorname{Re} \lambda)}{d \tau}\right\}_{\lambda=i w_{-}}=\operatorname{sign}\left\{\frac{-\sqrt{\left(b^{2}-a^{2}+2 d\right)^{2}-4\left(d^{2}-c^{2}\right)}}{\left\{\left(-w_{-}^{2}+\mathrm{d}\right)^{2}+\mathrm{a}^{2} w_{-}^{2}\right\}\left\{\mathrm{c}^{2}+\mathrm{b}^{2} w_{-}^{2}\right\}}\right\}$

Thus

$\left.\frac{d(\operatorname{Re} \lambda)}{d \tau}\right|_{\tau=\tau_{k}^{-}, w=w_{-}}<0$

Thus, the transferability conditions are satisfied.

\section{Description of the Numerical Method}

In this we follow the work $[3,4]$ to design a positivity preserving numerical method for solving model.

Let $N$ be any positive integer, and $h=T / N$. Let $t_{j}=j \cdot h$, for $j=0, \ldots, N$. Let $x^{j} \approx x\left(t_{j}\right)$ and $y^{j} \approx y\left(t_{j}\right)$. Then, model eqn. (1) can be approximated by the non-standard finite difference formula:

$$
\begin{aligned}
& \frac{x^{j+1}-x^{j}}{h}=-a_{1} x^{j+1}+a_{3}-a_{2} x_{\tau}^{j} y_{\tau}^{j}, j=0, \ldots, \mathrm{N}-1 \\
& \frac{y^{j+1}-y^{j}}{h}=b_{1} x^{j}-b_{2} y_{j+1}, j=0, \ldots, N-1
\end{aligned}
$$

from which we obtain the two relations:

$$
\begin{aligned}
& x^{j+1}=\frac{x^{j}+h\left(a_{3}-a_{2} x_{\tau}^{j} y_{\tau}^{j}\right)}{1+h a_{1}}, j=0, \ldots, N-1 \\
& y^{j+1}=\frac{y^{j}+h b_{1} x^{j}}{1+h b_{2}}, j=0, \ldots, N-1
\end{aligned}
$$

where, $x_{\tau}^{j}$ approximates $x\left(t_{j}-\tau\right)$ and $y_{\tau}^{j}$ approximates $y\left(t_{j-} \tau\right)$.

Let

$$
S=\left[\frac{\tau}{h}\right]
$$

then $\tau \approx S \cdot h$. Now, $x\left(t_{j}-\tau\right)$ can be approximated by $x\left(t_{j-S}\right)$ for $j \geq S$ and $y\left(t_{j}-\tau\right)$ can be approximated by $y\left(t_{j-S}\right)$ for $j \geq S$.

Then,

$$
y\left(t_{j}-\tau\right)= \begin{cases}\phi\left(t_{j}-\tau\right) & j<S \\ y^{j-s} & j \geq S\end{cases}
$$

and

$$
y\left(t_{j}-\tau\right)= \begin{cases}\phi\left(t_{j}-\tau\right) & j<S \\ y^{j-s} & j \geq S\end{cases}
$$

\section{Numerical Simulations}

In this, section we give some numerical simulations supporting our theoretical predictions. This present some examples and numerical simulations to verify our theoretical results proved in the previous using matlab program.

We consider the system:

$$
\begin{aligned}
& \frac{d x}{d t}=-0.1135 x(t)-1.4 x(t-\tau) y(t-\tau)+1.0023 \\
& \frac{d y}{d t}=0.22 x(t)-0.2972 y(t) .
\end{aligned}
$$

There is a positive equilibrium $\left(x^{*}, y^{*}\right)=(0.9302,0.6886)$.

Case I: For $\tau=0$. In this case, the numerical simulation (Figures 1 and 2) shows that both the glucose and insulin converge in finite time to their equilibrium values $x^{*}=0.9302$ and $y^{*}=0.6886$, respectively.

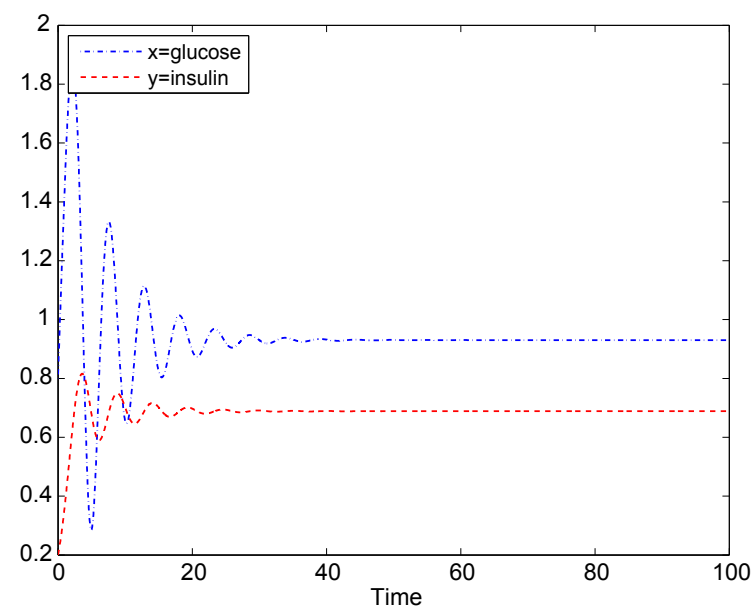

Figure 1: Glucose-Insulin dynamics for $\mathrm{T}=1.0738$.

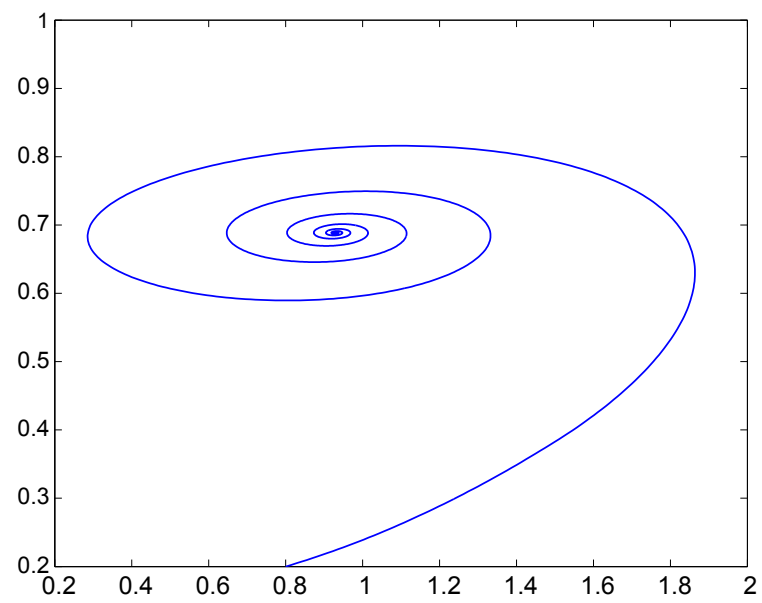

Figure 2: Phase plain of the Glucose-Insulin dynamics for $\mathrm{T}=1.0738$ 
Also in eqn. (9), i.e., the interior-equilibrium point $E_{1}$ is locally asymptotically stable when $\tau=0$ since

$$
\left(b_{1-} a_{2} x^{*}\right)^{2=0} 0.9022<4 b_{2}\left(a_{1+} a_{2} y^{*}\right)=1.4259 .
$$

Case II: For $\tau \neq 0$, by Theorem 1.2, there is a critical value $\tau_{0}=1.2738$. The computer simulations (Figures 1-4), show that $E_{1}$ is asymptotically stable when $\tau=1: 0738<\tau_{0=} 1.2738$. When $\tau$ passes through the critical value $\tau_{0}=1.2738, E_{1}$ loses its stability and a Hopf bifurcation occurs, i.e., a family of periodic solutions bifurcate from $E_{1}$. When $\tau>\tau_{0=} 1.2738, E_{1}$ is unstable (Figures 5 and 6).

\section{Conclusion}

Delay differential equations are an interesting form of differential equations, with many different applications, particularly in the biological and medical worlds. In this paper, a mathematical model has been proposed and analyzed to study the dynamics of glucose and insulin in the human body. Numerical simulations are carried out to demonstrate the results obtained. Appropriately determining the range of the delay based on physiology and clinical data is important in theoretical study. All the numerical results and graphs presented in the project were in agreement with those presented in

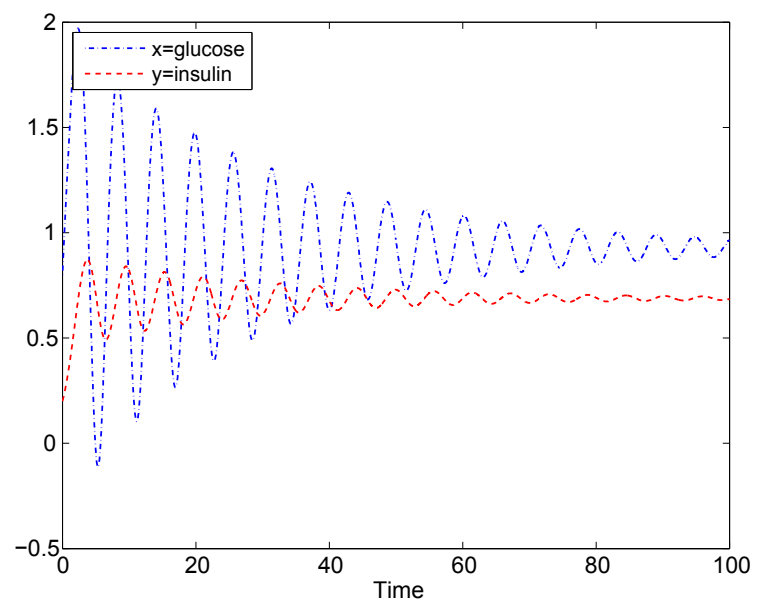

Figure 3: Glucose-Insulin dynamics for $\mathrm{T}=1.2738$

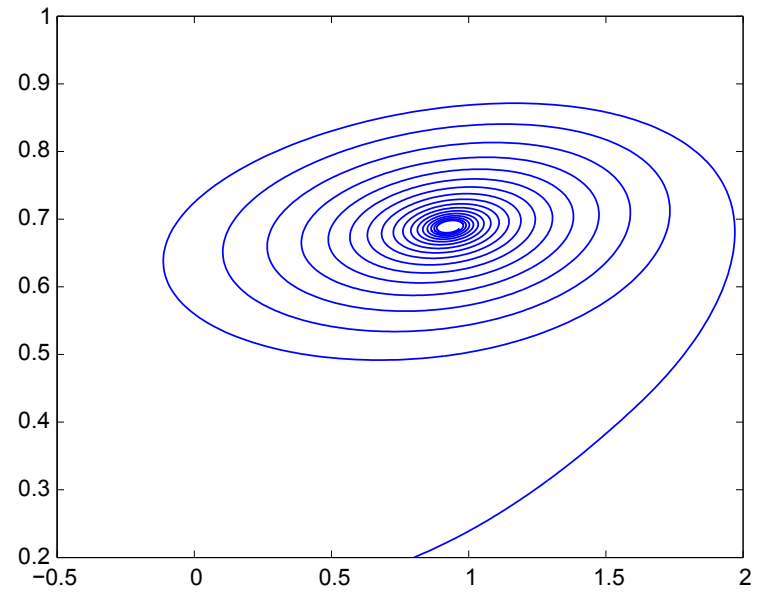

Figure 4: Phase plain of the Glucose-Insulin dynamics for $\mathrm{T}=1.2738$.

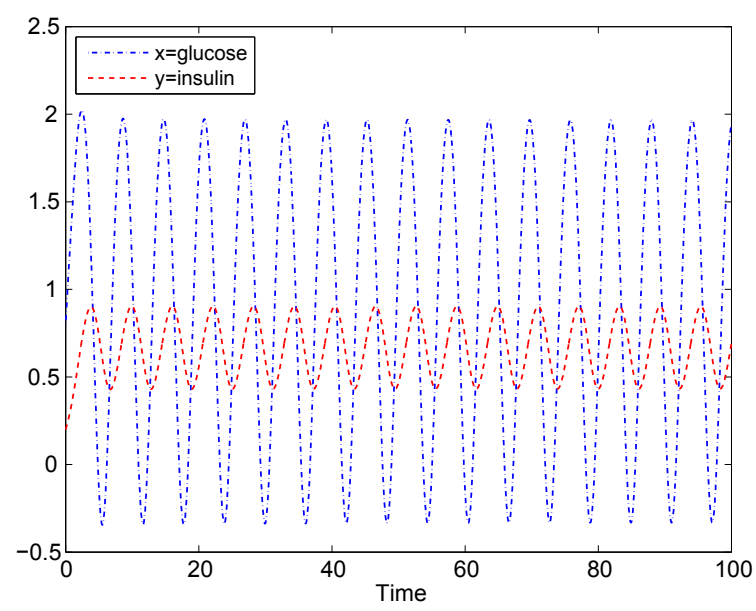

Figure 5: Glucose-Insulin dynamics for $\mathrm{T}=1.3738$

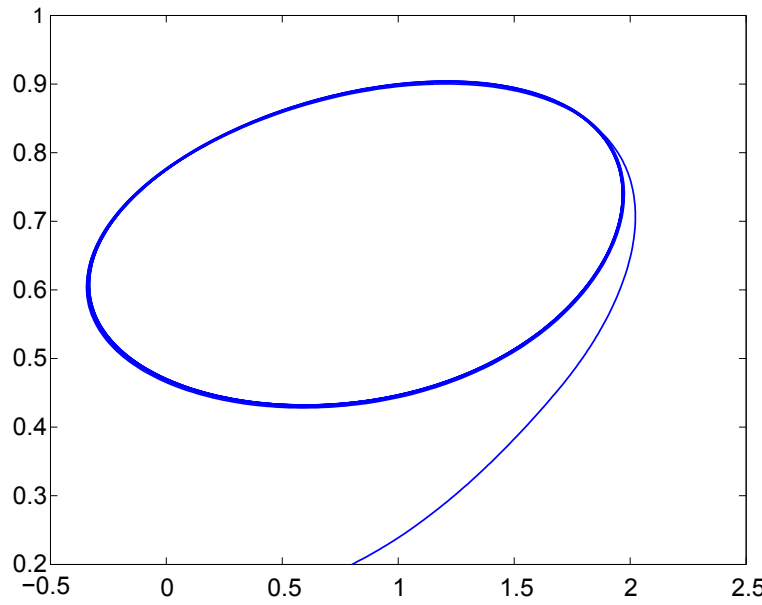

Figure 6: Phase plain of the Glucose-Insulin dynamics for $\mathrm{T}=1.3738$.

the relevant corresponding papers. Our results reveal the conditions on the parameters so that the periodic solution exist surrounding the interior equilibrium. It shows that $\tau_{0}$ is a critical value for the parameter $\tau$. Furthermore, the direction of Hopf bifurcation and the stability of bifurcated periodic solutions are investigated. From the above results, we conclude that the model is physiologically consistent and may be a useful tool for further research on diabetes.

\section{References}

1. Ajmera I, Swat M, Laibe C, Novre NL, Chelliah V (2013) The impact of mathematical modeling on the understanding of diabetes and related complications. CPT Pharmacometrics Syst Pharmacol 2: 1-14.

2. Balakrishnan NP, Rangaiah GP, Samavedham L (2011) Review and analysis of blood glucose (BG) models for type 1 diabetic patients. Ind Eng Chem Res 50: 12041-12066.

3. Bashier EBM, Patidar KC (2010) Fitted numerical methods for a system of first order delay differential equations. In: Ladde GS, Medhin NG, Peng C, Sambandham M editors. Proceedings of Neural, Parallel and Scientific Computations Dynamic Publishers Inc, 4: 48-53.

4. Bashier EBM, Patidar KC (2012) A Comparison of Numerical Methods for Systems of First Order Delay Differential Equations Arising in Biology, book chapter: A Treatise of Biological Models. Nova Publishers, pp: 75-96.

5. Bergman RN, Zaccaro DJ, Watanabe RM, Haffner SM, Saad MF, et al. (2003) 
Citation: Saber S, Bashier EBM, Alzahrani SM, Noaman IA (2018) A Mathematical Model of Glucose-Insulin Interaction with Time Delay. J Appl Computat Math 7: 416. doi: 10.4172/2168-9679.1000416

Page 5 of 5

Minimal Model Based Insulin Sensitivity Has Greater Heritability and a Different Genetic Basis Than Homeostasis Model Assessment or Fasting Insulin. J Diabetes 52: 2168-2174.

6. Cushing JM (1977) Integro-Differential Equations and Delay Models in Population Dynamics. Lecture Notes in Biomathematics.

7. Cushing JM, Saleem M (1982) A predator-prey model with age structure. J Math Biol 14: 231-250

8. Wang X, Yang J, Li X (2011) Dynamics of a Heroin Epidemic Model with Very Population. Appl Math 2: 732-738.

9. Hussain J, Zadeng D (2014) A mathematical model of glucose-insulin interaction. Sci Vis 14: 84-88.

10. Cushing JM, Saleem M (2003) A predator-prey model with age structure. J Math Biol 38: 449-458

11. Makroglou A, Jiaxu, Kuang $Y$ (2006) Mathematical models and software tools for the glucose-insulin regulatory system and diabetes: an overview. Appl Numer Math 56: 559-573.
12. Pedersen MG (2009) Contributions of mathematical modelling of beta cells to the understanding of beta-cell oscillations and insulin secretion. J Diabetes Sc Technol 3: 12-20.

13. Roy MSA, Parker RS (2007) Dynamic Modelling of Exercise Effects on Plasma Glucose and Insulin Levels. J Diabetes Sci Technol 1: 338-347.

14. Rubin AL (2015) Diabetes For Dummies, John Wiley and Sons Inc., 5th edition.

15. Sandhya , Kumar D (2011) Mathematical Model for Glucose-Insulin Regulatory System of Diabetes Mellitus. Advances in Applied Mathematical Biosciences 2. $39-46$.

16. Silber HE, Jauslin PM, Frey N, Gieschke R, Simonsson US, et al. (2007) An integrated model for glucose and insulin regulation in healthy volunteers and type 2 diabetic patients following intravenous glucose provocations. J Clin Pharmacol 47: 1159-1171.

17. Sthl F, Johansson R (2009) Diabetes mellitus modelling and short-term prediction based on blood glucose measurements. Mathematical Biosciences 217: $101-117$ 